\title{
Síndrome de fibromatosis hialina: reporte de dos casos de una misma familia
}

\author{
Hyaline fibromatosis syndrome: case report of two siblings
}

\author{
Estud. Diego Alejandro Rangel Rivera ${ }^{a}$, Dr. Víctor Clemente Mendoza Rojas ${ }^{b}$, \\ Dra. Claudia Janeth Uribe Pérezc y Dr. Gustavo Adolfo Contreras-García ${ }^{d}$
}

\section{RESUMEN}

El síndrome de fibromatosis hialina es una enfermedad autosómica recesiva rara, que se caracteriza por la presencia de contractura y dolorarticular, placas y nóduloshiperpigmentados e hipertrofia gingival, producto de la acumulación de un material amorfo hialino similar al colágeno tipo VIen diferentes tejidos. Esta enfermedad incluye el síndrome de hialinosis sistémica y la fibromatosis hialina juvenil, dos entidades que, durante años, fueron consideradas de manera separada; sin embargo, las características clínicas y la edad de presentación se superponen. Además, ha sido documentado que la causa de ambas entidades se localiza en un mismo gen. Se presentan dos casos de hermanas de una misma familia colombiana afectadas por la enfermedad.

Palabras clave: síndrome de fibromatosis hialina, contractura articular, hipertrofia gingival.

\section{ABSTRACT}

Hyaline fibromatosis syndrome is a rare autosomal recessive disease characterized by the presence of contracture and joint pain, hyperpigmented plaques and nodules and gingival hypertrophy. These findings are the result of the accumulation of a hyaline amorphous material similar to collagen type VI in different tissues. This syndrome includes systemic hyalinosis and juvenile hyaline fibromatosis, two entities that for years were considered separately. However, it has been documented that the cause of both entities is located in the same gene and the clinical features and age of presentation are overlapped. In this study two cases of sisters from a same colombian family affected by the disease are presented.

Key words: hyaline fibromatosis syndrome, joint contracture, gingival hypertrophy.

a. Grupo de investigación en Genética Humana. Escuela de Medicina. Universidad Industrial de Santander.

b. Departamento de Pediatría. Universidad Industrial de Santander.

c. Facultad de Ciencias de la Salud, programa de Medicina. Universidad Autónoma de Bucaramanga.

d. Grupo de investigación en Genética Humana. Escuela de Medicina. Universidad Industrial de Santander.

Bucaramanga. Colombia.

Correspondencia:

Estud. Diego Alejandro Rangel Rivera, dar_2333@hotmail.com

Financiamiento: ninguno.

Conflicto de intereses: ninguno que declarar.

Recibido: 2-12-2014

Aceptado: 27-3-2015 http:/ / dx.doi.org/10.5546/aap.2015.e264

\section{INTRODUCCIÓN}

El síndrome de fibromatosis hialina (SFH) (Hyaline Fibromatosis Syndrome, HFS) (OMIM \# 228600) se considera un trastorno autosómico recesivo que comprende dos entidades generadas por mutaciones en el gen del receptor 2 de la toxina del ántrax (anthraxtoxin receptor 2 gene, ANTRX2) localizado en la región cromosómica $4 \mathrm{q} 21 .{ }^{1}$ la hialinosis sistémica infantil (HSI) y la fibromatosis hialina juvenil (FHJ).

El cuadro clínico se caracteriza por contractura progresiva de articulaciones, llanto con la movilización articular, pápulas y nódulos hiperpigmentados (especialmente en la cara y el cuello), hipertrofia gingival, infecciones frecuentes y diarrea con presencia de enteropatía perdedora de proteínas., ${ }^{2,3}$ A pesar de existir alrededor de 150 casos reportados de SFH, no se ha determinado aun completamente su fisiopatología. El objetivo del presente artículo es comunicar dos casos de SFH pertenecientes a una misma familia.

\section{PRESENTACIÓN DE CASOS \\ Caso 1}

Paciente femenina de 26 meses de edad, procedente de Arauca, Colombia. Padre de 36 años y madre de 39 años. Producto de primera gestación, sin antecedentes patológicos familiares ni consanguinidad parental. Embarazo normal sin exposición a teratógenos, ecografías sin alteraciones y parto a las 37 semanas sin complicaciones. A los 4 meses de vida, se evidenció limitación simultánea en la movilidad articular de manera generalizada. A los 6 meses, presentó una lesión eritematosa en el maxilar inferior, que progresó con el tiempo y aumentó de tamaño.

Al momento del examen físico, se documentó la presencia de plagiocefalia y facies tosca (Figura 1. A). Además, presentó aumento de párpados, labios, maxilar, fosas nasales y pabellón auricular 
derecho; cuello corto y ancho con limitación para los movimientos; limitación de la movilidad articular de manera simétrica y simultánea en las extremidades, camptodactilia y hoyuelos en las manos (Figura 1. B). La biopsia de las lesiones de piel demostró características compatibles con SFH (Figura 2. A-B). La paciente falleció a los 3 años de vida debido a neumonía adquirida en la comunidad.

\section{Caso 2}

Paciente de sexo femenino, de 12 meses de edad, hermana del caso 1 descrito, también procedente de Arauca, Colombia. Derivada por presencia de contractura articular generalizada desde los 6 meses de edad, asociada a un aumento del tamaño y pigmentación de la piel adyacente a las articulaciones interfalángicas

Figura 1. A. Facies tosca, aumento de párpados, labios, maxilar y fosas nasales. Cuello corto y ancho. B. Se aprecia camptodactilia y hoyuelos en las manos

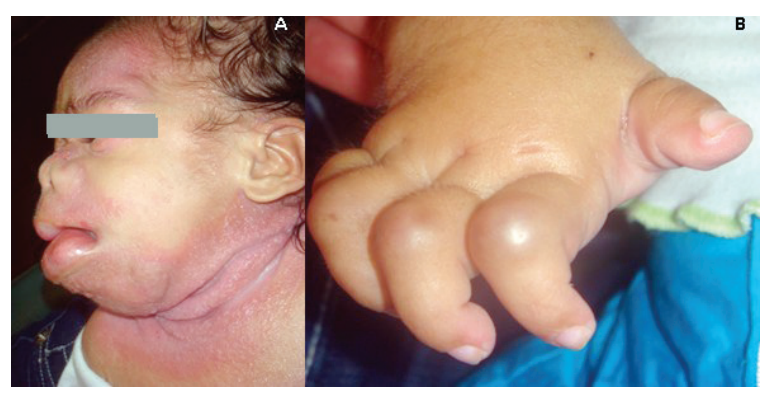

FIGURA 2. A. Se observa epidermis con zonas de adelgazamiento y depósito de material eosinófilo en la dermis, que altera la estructura de los anexos cutáneos. B. A mayor aumento, material eosinofílico depositado en la dermis, más evidente alrededor de los vasos sanguíneos, los cuales se aprecian dilatados ( $A$ y $B$, tinción $H E ; A$ : $4 X ; B$ : 10X)

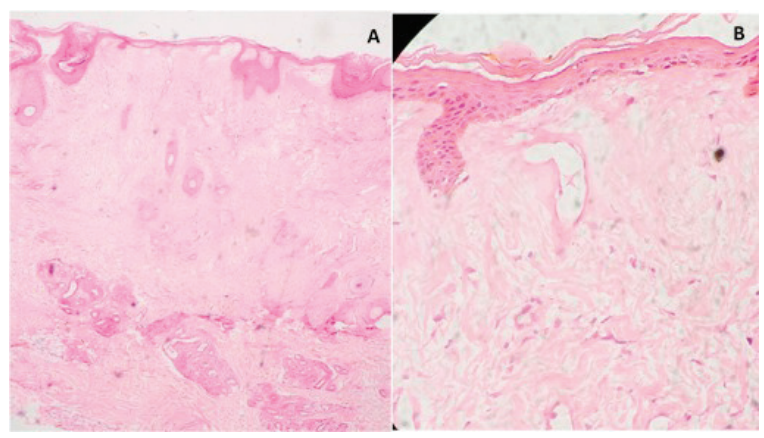

tanto proximales como distales. Posteriormente, presentó deformidad a nivel gingival y labial.

Producto de tercera gestación, con ecografías prenatales normales y sin exposición a teratógenos. Parto vaginal eutócico a las 39 semanas, sin complicaciones; recién nacido pequeño para la edad gestacional (2160 g). Antecedente de sepsis grave con foco pulmonar a los 6 meses edad.

Presentaba un retardo pondoestatural con un peso de $5100 \mathrm{~g}(-48 \% \mathrm{P} / \mathrm{E})$ y talla de $66 \mathrm{~cm}$ (2,5 DE). Se evidenció un aumento del tamaño a nivel labial superior, asociado a hiperplasia gingival con paladar normal y narinas antevertidas (Figura 3. A-B). Se observaron placas eritematosas y descamativas en ambos pabellones auriculares (Figura 3. C) y lesiones de iguales características en la región posterior de la base del cuello (Figura 3. A). Presentaba 2 pápulas hiperpigmentadas: la primera localizada en la región posterior del hemitórax superior; la segunda, en la región posterior del muslo derecho (Figura 4. A-B). Limitación en la movilidad de todas las articulaciones tanto de los miembros superiores como de los miembros inferiores. Coloración violácea de la piel de las articulaciones interfanlángicas proximales y distales (Figura 4. C), al igual que en la región adyacente al olécranon y al maléolo externo (Figura 4. D-E). En el examen neurológico y cardiopulmonar, no se observaron alteraciones. Actualmente, se halla en seguimiento en el Servicio de Genética, con presencia de cuadros infecciosos graves repetidos y rápida progresión de su patología.

\section{DISCUSIÓN}

El SFH es una enfermedad autosómica recesiva rara. Han sido documentados alrededor de 80 casos de FHJ y 70 casos de HSI. En Latinoamérica,

Figura 3. A. Facies tosca con narinas antevertidas. B. Hiperplasia gingival con paladar normal. C. Placas eritematosas descamativas localizadas de manera bilateral en pabellones auriculares

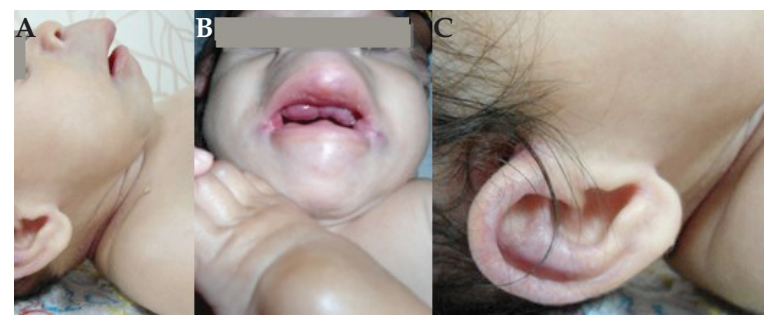


se han descrito un total de 17 casos de SFH, 10 de los cuales se han reportado en Brasil. De acuerdo con nuestro conocimiento, estos son los primeros dos casos documentados en Colombia.

El término SFH fue propuesto por Nofal y col., ${ }^{4}$ para agrupar el síndrome de HSI y el síndrome de FHJ. Para esta sugerencia, el grupo se basó en dos aspectos clave: el primero consiste en que ambas patologías tienen un mismo origen genético con mutaciones en el gen del ANTRX2; en segundo lugar, existe dificultad para clasificar a un paciente en uno $u$ otro síndrome porque sus criterios clínicos, histopatológicos y genéticos se superponen. ${ }^{4-6}$ Además, el término hialinosis sistémica no se utiliza puesto que se excluirían los casos de enfermedad localizada.

Los niños con SFH pueden presentar signos desde el nacimiento, dentro de los primeros meses o años de vida, especialmente antes del cuarto año. Las manifestaciones se caracterizan por lesiones pigmentadas difusas, nódulos pequeños hiperpigmentados en las caderas, la región perianal, la región auricular, el cuero cabelludo, la cara y el mentón, puente nasal deprimido, hiperplasia gingival, compromiso

Figura 4. A. Pápula grisácea de 0,5 $\times 0,3 \mathrm{~cm}$ en la región escapular izquierda. B. Pápula café de 0,6 $\times 0,4 \mathrm{~cm}$ en la región posterior del muslo derecho. C. Coloración violácea yuxtaarticular en la mano izquierda. D. Coloración violácea en la región del olécranon izquierdo.

E. Coloración violácea en el maléolo externo derecho

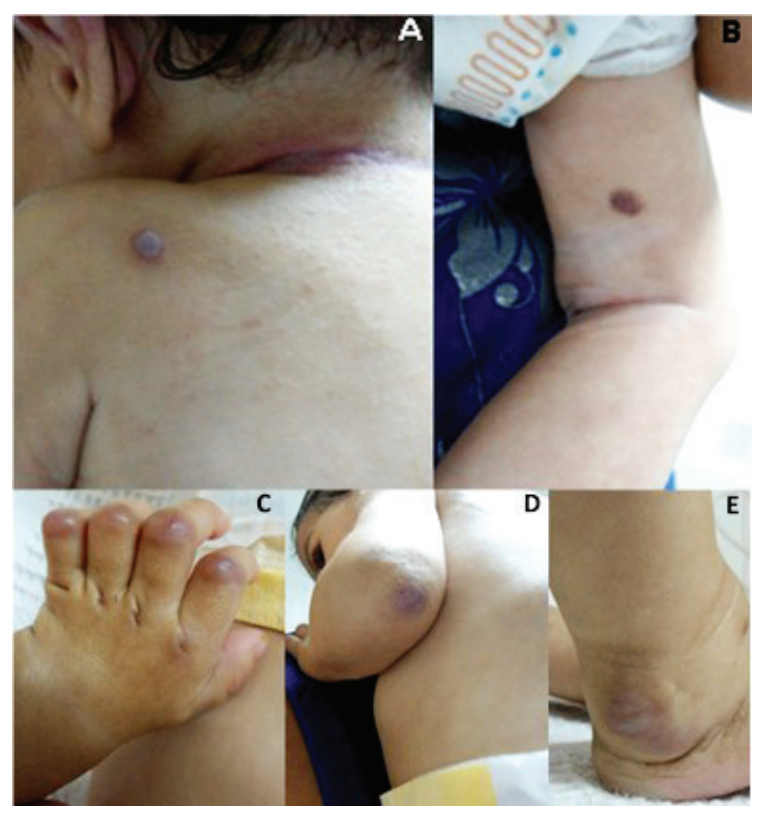

articular y retardo del crecimiento asociado a infecciones frecuentes, como en los dos pacientes presentados. ${ }^{2}$ La afectación articular suele ser progresiva y simétrica en las extremidades. ${ }^{3,5}$ Estos niños pueden morir dentro de sus primeros meses de vida asociado a infecciones pulmonares y diarrea o pueden llegar hasta la edad adulta., ${ }^{7,8}$ Los pacientes con SFH tienen un neurodesarrollo normal. ${ }^{5,6}$

Nofal y col., ${ }^{4}$ propusieron una clasificación para los pacientes con SFH en grados leve, moderado y grave, y posteriormente, Denadai y col., ${ }^{5}$ la modificaron y agregaron el grupo letal. El grado leve corresponde a los pacientes con hiperplasia gingival y compromiso cutáneo; el grado moderado, a los que, además de lo anterior, presentan compromiso articular o lesiones óseas; el grado grave incluye a los pacientes que tienen también compromiso en otros órganos y presentan síntomas como diarreas persistentes e infecciones pulmonares a repetición; y la forma letal describe a los pacientes que presentan falla orgánica o septicemia. Nuestros casos se clasifican dentro de este último grupo.

A pesar de que la SFH es consecuencia de mutaciones en el gen del ANTRX2, la fisiopatología no es completamente entendida. E1 ANTRX2 codifica para una proteína transmembrana que presenta unión con colágeno tipo IV y laminina, y desempeña un papel esencial durante la formación de la matriz de colágeno extracelular y la morfogénesis capilar. Se considera que los síntomas presentados en la enfermedad son consecuencia de la acumulación perivascular de material hialino en los diversos órganos afectados.

Para el diagnóstico, debe tenerse en cuenta el cuadro clínico compatible, especialmente el compromiso articular, las lesiones en la piel y la hiperplasia gingival. ${ }^{9}$ En estudios radiológicos, suelen observarse lesiones osteolíticas en huesos largos y falanges distales. ${ }^{8,10}$ El estudio molecular confirma el diagnóstico a través de la búsqueda de mutaciones del gen del ANTRX2, de las cuales se han documentado, por lo menos, 28 mutaciones diferentes en la literatura. ${ }^{11,12}$ Se deberá realizar el diagnóstico diferencial con otras patologías, como la miofibromatosis congénita generalizada, lipogranulomatosis de Farber, mucopolisacaridosis y el síndrome de Winchester. ${ }^{5,6}$

Dentro de las características histopatológicas, se destaca la presencia de material eosinofílico amorfo en la dermis. ${ }^{2,5}$ Este material suele 
localizarse en mayor densidad en el área perivascular, como fue observado en uno de nuestros pacientes. ${ }^{5}$ Asimismo, se ha comunicado un aumento de la actividad fibroblástica en las biopsias de piel de estos pacientes. ${ }^{4}$

En la actualidad, no existe un tratamiento específico para esta enfermedad progresiva, por lo que toma relevancia la asesoría genética. Para el tratamiento de las lesiones orales y dermatológicas, la escisión quirúrgica se convierte en el pilar del manejo. ${ }^{6,13}$ La capsulotomía articular puede brindar un alivio temporal del dolor. ${ }^{5}$ Asimismo, el uso de D-penicilamina se ha asociado con la mejoría de la movilidad y flexibilidad articular. ${ }^{6}$ Recientemente, se usó el octreotide por muy corto tiempo para el manejo de un paciente con enteropatía perdedora de proteínas, pero no se asoció a cambios en el nivel de albúmina. ${ }^{12}$

Se ha demostrado que se puede obtener un aumento en la cantidad de ANTRX2 en fibroblastos de pacientes con esta patología a través de inhibidores de proteosomas. ${ }^{14}$ Ello tendría un potencial efecto terapéutico porque la disminución de la actividad por retención de la proteína en el retículo endoplasmático puede aumentar la cantidad de proteína en la membrana plasmática con la posibilidad de realizar su función normal. ${ }^{15}$

\section{CONCLUSIÓN}

El SFH es una entidad muy rara, sin embargo, el equipo médico debe tener la capacidad para realizar un enfoque diagnóstico adecuado a través de la utilización de las herramientas clínicas, histopatológicas y moleculares. El manejo de esta patología requiere un trabajo multidisciplinario para abordar las diferentes complicaciones que pueden derivar de la enfermedad. Además, se debe brindar asesoría genética a la familia, teniendo en cuenta que es una patología autosómica recesiva, por lo que el riesgo de recurrencia corresponde al $25 \%$.

\section{REFERENCIAS}

1. Dowling O, Difeo A, Ramírez MC, Tukel T, et al. Mutations in capillary morphogenesis gene-2 result in the allelic disorders juvenile hyaline fibromatosis and infantile systemic hyalinosis. Am J Hum Genet 2003;73(4):957-66.

2. Shin HT, Paller A, Hoganson G, Willner JP, et al. Infantile systemic hyalinosis. J Am Acad Dermatol 2004;50(2 Suppl):S61-4.

3. Shieh JT, Swidler P, Martignetti JA, Ramírez MC, et al. Systemic hyalinosis: a distinctive early childhood-onset disorder characterized by mutations in the anthrax toxin receptor 2 gene (ANTRX2). Pediatrics 2006;118(5):e1485-92.

4. Nofal A, Sanad M, Assaf M, Nofal E, et al. Juvenile hyaline fibromatosis and infantile systemic hyalinosis: a unifying term and a proposed grading system. I Am Acad Dermatol 2009;61(4):695-700.

5. Denadai R, Raposo-Amaral CE, Bertola D, Kim C, et al. Identification of 2 novel ANTXR2 mutations in patients with hyaline fibromatosis syndrome and proposal of a modified grading system. Am J Med Genet A 2012;158A(4):732-42.

6. Urbina F, Sazunic I, Murray G. Infantile systemichyalinosis or juvenile hyaline fibromatosis? Pediatr Dermatol 2004;21(2):154-9.

7. Lindvall LE, Kormeili T, ChenE, Ramírez MC, etal. Infantile systemichyalinosis: Case report and review of the literature. J Am Acad Dermatol 2008;58(2):303-7.

8. SlimaniS,Haddouche A,HaidS, Ladjouze-Rezig A.Juvenile hyaline fibromatosis: focus on radiographic features in adulthood. Rheumatol Int 2011;31(2):273-6.

9. M VR, J PG, Arava S, B NM, et al. Juvenile Hyaline Fibromatosis (JHF): a rare case with recurrence. JClin Diagn Res 2014;8(2):161-2.

10. Yoo SY, Kim JH, Kang HS, Hwang YS, et al. Clinical and imaging findings of systemic hyalinosis: two cases presenting with congenital arthrogryposis. Skeletal Radiol 2010;39(6):589-93.

11. El-Kamah GY, Fong K, El-Ruby M, AfifiHH, et al.Spectrum of mutations in the ANTXR2 (CMG2) gene in infantile systemic hyalinosis and juvenile hyaline fibromatosis. $\mathrm{Br}$ J Dermatol 2010;163(1):213-5.

12. AlSinani S, Al Murshedy F, Abdwani R. Infantile systemic hyalinosis: a case report with a novel mutation. Oman Med J 2013;28(1):53-5.

13. Jaouad IC, Guaoua S, Hajjioui A, Sefiani A. Hyaline fibromatosis syndrome with mutation c.1074delT of the CMG2 gene: a case report. J Med Case Rep 2014;8:291.

14. Deuquet J, Lausch E, Guex N, Abrami L, et al. Hyaline fibromatosis syndrome inducing mutations in the ectodomain of anthrax toxin receptor 2 can be rescued by proteasome inhibitors. EMBO Mol Med 2011;3(4):208-21.

15. Deuquet J, Abrami L, Difeo A, Ramírez MC, et al. Systemic hyalinosis mutations in the CMG2 ectodomain leading to loss of function through retention in the endoplasmic reticulum. Hum Mutat 2009;30(4):583-9. 